\title{
SIGN CHANGES OF THE LIOUVILLE FUNCTION ON QUADRATICS
}

\author{
PETER BORWEIN, STEPHEN K.K. CHOI AND HIMADRI GANGULI
}

Abstract. Let $\lambda(n)$ denote the Liouville function. Complementary to the prime number theorem, Chowla conjectured that

Conjecture (Chowla).

$$
\sum_{n \leq x} \lambda(f(n))=o(x)
$$

for any polynomial $f(x)$ with integer coefficients which is not of form $b g(x)^{2}$.

The prime number theorem is equivalent to 0.1 when $f(x)=x$. Chowla's conjecture is proved for linear functions but for the degree greater than 1 , the conjecture seems to be extremely hard and still remains wide open. One can consider a weaker form of Chowla's conjecture, namely,

Conjecture 1 (Cassaigne, et al). If $f(x) \in \mathbb{Z}[x]$ and is not in the form of $b g^{2}(x)$ for some $g(x) \in \mathbb{Z}[x]$, then $\lambda(f(n))$ changes sign infinitely often.

Clearly, Chowla's conjecture implies Conjecture 1. Although it is weaker, Conjecture 1 is still wide open for polynomials of degree $>1$. In this article, we study Conjecture 1 for the quadratic polynomials. One of our main theorems is

Theorem 1. Let $f(x)=a x^{2}+b x+c$ with $a>0$ and $l$ be a positive integer such that al is not a perfect square. Then if the equation $f(n)=l^{2}$ has one solution $\left(n_{0}, m_{0}\right) \in \mathbb{Z}^{2}$, then it has infinitely many positive solutions $(n, m) \in \mathbb{N}^{2}$.

As a direct consequence of Theorem 1, we prove

Theorem 2. Let $f(x)=a x^{2}+b x+c$ with $a \in \mathbb{N}$ and $b, c \in \mathbb{Z}$. Let

$$
A_{0}=\left[\frac{|b|+(|D|+1) / 2}{2 a}\right]+1 .
$$

Then the binary sequence $\{\lambda(f(n))\}_{n=A_{0}}^{\infty}$ is either a constant sequence or it changes sign infinitely often.

Some partial results of Conjecture 1 for quadratic polynomials are also proved by using Theorem 1 .

\section{INTRODUCTION}

Let $\lambda(n)$ denote the Liouville function, i.e, $\lambda(n)=(-1)^{\Omega(n)}$, where $\Omega(n)$ denotes the number of prime factors of $n$ counted with multiplicity. Alternatively, $\lambda(n)$ is

Date: November 14, 2018.

1991 Mathematics Subject Classification. Primary 11N60, 11B83, $11 D 09$.

Key words and phrases. Liouville Function, Chowla's Conjecture, Prime Number Theorem, Binary Sequences, Changes sign Infinitely Often, Quadratic Polynomials, Pell Equations.

Research of first and second authors are supported by NSERC of Canada. 
the completely multiplicative function defined by $\lambda(p)=-1$ for each prime. Let $\zeta(s)$ denote the Riemann zeta function, defined for complex $s$ with $\Re(s)>1$ by

$$
\zeta(s):=\sum_{n=1}^{\infty} \frac{1}{n^{s}}=\prod_{p}\left(1-\frac{1}{p^{s}}\right)^{-1}
$$

where the product is over all prime numbers $p$. Thus

$$
\frac{\zeta(2 s)}{\zeta(s)}=\prod_{p}\left(1+\frac{1}{p^{s}}\right)^{-1}=\prod_{p}\left(1-\frac{\lambda(p)}{p^{s}}\right)^{-1}=\sum_{n=1}^{\infty} \frac{\lambda(n)}{n^{s}} .
$$

Let $L(x)$ denote the average of the values of $\lambda(n)$ up to $x$,

$$
L(x):=\sum_{1 \leq n \leq x} \lambda(n)
$$

so that $L(x)$ records the difference of the number of positive integers up to $x$ with an even number of prime factors (counted with multiplicity) and those with an odd number. Pólya in 1919 showed in [10] that the Riemann Hypothesis, i.e., every non-trivial zeros of $\zeta(s)$ are on the critical line $\Re(s)=1 / 2$, will follow if $L(x)$ does not change sign for sufficiently large $n$. There is a vast amount of literature about the study of the sign change of $L(x)$. Until 1958, Haselgrove proved that $L(x)$ changes sign infinitely often in [4. For more discussion about this problem, we refer the reader to 1$]$.

It is well known that the prime number theorem is equivalent to

$$
L(x)=\sum_{n \leq x} \lambda(n)=o(x) .
$$

In fact, the prime number theorem is equivalent to that fact that $\zeta(s) \neq 0$ on the vertical line $\Re(s)=1$; and this is equivalent to (1.2) in view of (1.1). Complementary to the prime number theorem, Chowla 3 made the following conjecture

Conjecture 1 (Chowla). Let $f(x) \in \mathbb{Z}[x]$ be any polynomial which is not of form $b g^{2}(x)$ for some $b \neq 0, g(x) \in \mathbb{Z}[x]$. Then

$$
\sum_{n \leq x} \lambda(f(n))=o(x)
$$

Clearly, Chowla's conjecture is equivalent to the prime number theorem when $f(x)=x$. For polynomials of degree $>1$, Chowla's conjecture seems to be extremely hard and still remains wide open. One can consider a weaker form of Chowla's conjecture, namely,

Conjecture 2 (Cassaigne et al.). If $f(x) \in \mathbb{Z}[x]$ is not of form $b g^{2}(x)$, then $\lambda(f(n))$ changes sign infinitely often.

Clearly, Chowla's conjecture implies Conjecture 2, In fact, suppose it is not true, i.e., there is $n_{0}$ such that $\lambda(f(n))=\epsilon$ for all $n \geq n_{0}$ where $\epsilon$ is either -1 or +1 . Then it follows that

$$
\sum_{n \leq x} \lambda(f(n))=\epsilon x+O(1)
$$

which contradicts (1.3).

Although it is weaker, Conjecture 2 is still wide open for polynomials of degree $>1$. In [2, Conjecture2 for special polynomials have been studied and some partial results are proved. 
Theorem 1.1 (Cassaigne et al.). Let $f(n)=\left(a n+b_{1}\right)\left(a n+b_{2}\right) \ldots\left(a n+b_{k}\right)$ where $a, k \in \mathbb{N}, b_{1}, \ldots, b_{k}$ are distinct integers with $b_{1} \equiv \ldots \equiv b_{k}(\bmod a)$ then $\lambda(f(n))$ changes sign infinitely often.

Proof. This is Corollary 2 in [2].

For certain quadratic polynomials, they proved

Theorem 1.2 (Cassaigne et al.). If $f(n)=(n+a)(b n+c)$ where $a, c \in \mathbb{Z}, b \in$ $\mathbb{N}, a b \neq c$ then $\lambda(f(n))$ changes sign infinitely often.

Proof. This is Theorem 4 in [2].

Theorem 1.3 (Cassaigne et al.). Let $a \in \mathbb{N}, b, c \in \mathbb{Z}$, and write $f(n)=a n^{2}+b n+c$, $D=b^{2}-4 a c$. Assume that $a, b$ and $c$ satisfy the following conditions :

(i) $2 a \mid b$,

(ii) $D<0$,

(iii) there is a positive integer $k$ with

$$
\lambda\left(-\frac{D}{4} k^{2}+1\right)=-1
$$

Then $\lambda(f(n))$ changes sign infinitely often.

Proof. This is Theorem 3 in [2].

In this article, we continue to study Conjecture 2 for the quadratic case. One of our main results is Theorem 2.2 below. By Theorem 2.2 in order to show that the sequence $\{\lambda(f(n))\}_{n=1}^{\infty}$ changes sign infinitely often, we only need find one pair of large integers $n_{1}$ and $n_{2}$ such that $\lambda\left(f\left(n_{1}\right)\right) \neq \lambda\left(f\left(n_{2}\right)\right)$. This will make the conjecture much easier to handle. Some partial results from Theorem 2.2 are also deduced in the next section.

\section{Main Results}

Conjecture 2 for the linear polynomial is easily solved by the following result.

Theorem 2.1. Let $P:=\{n \in \mathbb{N}: \lambda(n)=+1\}$ and $N:=\{n \in \mathbb{N}: \lambda(n)=-1\}$. Then both $P$ and $N$ cannot contain infinite arithmetic progression. In particular, $\lambda(a n+b)$ changes sign infinitely often in $n$.

Proof. We claim that both $\mathrm{P}$ and $\mathrm{N}$ cannot contain any infinite arithmetic progression. Suppose not and there are an $n_{0}$ and $l$ such that

$$
\lambda\left(n_{0}+l k\right)=\lambda\left(n_{0}\right)
$$

for $k=0,1,2, \ldots$ Pick a prime $p$ which is of the form $l m+1$. Now put $k=m n_{0}$ and consider

$$
\lambda\left(n_{0}+l k\right)=\lambda\left(n_{0}+l m n_{0}\right)=\lambda\left(n_{0}\right) \lambda(l m+1)=\lambda\left(n_{0}\right) \lambda(p)=-\lambda\left(n_{0}\right) .
$$

This contradicts (1). Hence our claim is attained.

One of the main results in this paper is the following theorem.

Theorem 2.2. Let $f(x)=a x^{2}+b x+c$ with $a>0$ and $l$ be $a$ positive integer such that al is not a perfect square. Then if the equation $f(n)=l m^{2}$ has one solution $\left(n_{0}, m_{0}\right) \in \mathbb{Z}^{2}$, then it has infinitely many positive solution $(n, m) \in \mathbb{N}^{2}$. 
Proof. Let $D=b^{2}-4 a c$ be the discriminant of $f(x)$. By solving the quadratic equation

$$
a n^{2}+b n+c=l m^{2},
$$

for $n$ we get

$$
n_{0}=\frac{-b \pm \sqrt{b^{2}-4 a\left(c-l m_{0}^{2}\right)}}{2 a}=\frac{-b \pm \sqrt{D+4 a l m_{0}^{2}}}{2 a} .
$$

It follows that $D+4 a l m_{0}^{2}=t_{0}^{2}$ for some integer $t_{0}$. By choosing a suitable sign of $t_{0}$, we may assume

$$
t_{0} \equiv b \quad(\bmod 2 a), \quad \text { and } \quad n_{0}=\frac{-b+t_{0}}{2 a} \in \mathbb{Z} .
$$

This leads us to consider the diophantine equation

$$
t^{2}=4 a l m^{2}+D \text {. }
$$

Suppose that $\left(t_{0}, m_{0}\right)$ and $(t, m)$ are solutions of (2.4). Then we have

$$
t^{2}=4 a l m^{2}+D
$$

and

$$
t_{0}^{2}=4 a l m_{0}^{2}+D
$$

Subtracting the above two equations, we get

$$
\left(t-t_{0}\right)\left(t+t_{0}\right)=l\left(m-m_{0}\right)\left(4 a m+4 a m_{0}\right) .
$$

We now let $s$ and $r$ be

$$
r\left(m-m_{0}\right)=2 a s\left(t+t_{0}\right), 2 a s\left(4 a l m+4 a l m_{0}\right)=r\left(t-t_{0}\right) .
$$

By eliminating the terms $t$ and $m$ respectively in (2.6), we get

$$
\left(r^{2}-16 a^{3} l s^{2}\right) m=r^{2} m_{0}+16 a^{3} l s^{2} m_{0}+4 a r s t_{0}
$$

and

$$
\left(r^{2}-16 a^{3} l s^{2}\right) t=r^{2} t_{0}+16 a^{2} l s^{2} m_{0}+16 a^{3} s^{2} l t_{0} .
$$

Note that by our assumption, $16 a^{3} l$ is not a perfect square. So the Pell equation

$$
r^{2}-16 a^{3} l s^{2}=1
$$

always have infinitely many solutions $(r, s) \in \mathbb{Z}^{2}$, and for each solution $(r, s)$ of the Pell equation (2.9) gives integers $m$ and $t$ through (2.7) and (2.8) such that

$$
m=r^{2} m_{0}+16 a^{3} l s^{2} m_{0}+4 a r s t_{0}
$$

and

$$
t=r^{2} t_{0}+16 a^{2} l s r m_{0}+16 a^{3} s^{2} l t_{0} .
$$

One can easily verify that if $(r, s) \neq( \pm 1,0)$ then these $m$ and $t$ satisfy the equations (2.6) and hence satisfy equation (2.4). Note that $r^{2} \equiv 1(\bmod 2 a)$ and $r\left(m-m_{0}\right) \equiv$ $0(\bmod 2 a)$. Hence we have $m \equiv m_{0}(\bmod 2 a)$ and $t \equiv t_{0}(\bmod 2 a)$ by (2.6). Since there are infinitely many solutions $(r, s) \in \mathbb{Z}^{2}$ of the Pell equation (2.9) and these will give infinitely many solutions $(m, t) \in \mathbb{Z}^{2}$ of the equation (2.6). In particular, there are infinite many positive integers $t$ such that $t \equiv t_{0}(\bmod 2 a)$ and

$$
n=\frac{-b+\sqrt{D+4 a l m^{2}}}{2 a}=\frac{-b+t}{2 a}
$$


to be a positive integer by (2.3). Therefore, there are infinitely many positive solutions $(n, m) \in \mathbb{N}^{2}$ of (2.2). This completes the proof of the theorem.

It is worth to mention that one should not expect Theorem 2.2 is true for polynomials of higher degree because there may only have finitely many integer solutions by Siegel's theorem on integral points in [12].

In view of Theorem 2.2, to determine the conjecture is true for a given quadratic polynomial $f(x)$, we only need to find one pair of positive integers $n_{1}$ and $n_{2}$ such that $\lambda\left(f\left(n_{1}\right)\right) \neq \lambda\left(f\left(n_{2}\right)\right)$. This gives us the following theorem.

Theorem 2.3. Let $f(x)=a x^{2}+b x+c$ with $a \in \mathbb{N}$ and $b, c \in \mathbb{Z}$. Let

$$
A_{0}=\left[\frac{|b|+(|D|+1) / 2}{2 a}\right]+1 \text {. }
$$

Then the binary sequence $\{\lambda(f(n))\}_{n=A_{0}}^{\infty}$ is either a constant sequence or it changes sign infinitely often.

Proof. Suppose $\{\lambda(f(n))\}_{n=A_{0}}^{\infty}$ is not a constant sequence. Then there are positive integers $n_{1} \neq n_{2} \geq A_{0}$ such that $\lambda\left(f\left(n_{1}\right)\right) \neq \lambda\left(f\left(n_{2}\right)\right)$. Hence there are positive integers $l_{1}, l_{2}$ and $m_{1}, m_{2}$ such that

$$
\lambda\left(l_{1}\right)=+1, \quad \text { and } \quad \lambda\left(l_{2}\right)=-1,
$$

and

$$
f\left(n_{1}\right)=l_{1} m_{1}^{2}, \quad \text { and } \quad f\left(n_{2}\right)=l_{2} m_{2}^{2} .
$$

We claim that $a l_{1}$ and $a l_{2}$ are not perfect squares. If $a l_{j}=N^{2}$ is a perfect square, then the diophantine equation $t^{2}=D+4 a l_{j} m^{2}$ has only finitely many solutions $(t, m)$. In fact, since $\left(t_{j}-2 N m_{j}\right)\left(t_{j}+2 N m_{j}\right)=D$, so there is $d \neq 0$ such that $t_{j}+2 N m_{j}=D / d$ and $t_{j}-2 N m_{j}=d$. It follows that $2 t_{j}=D / d+d$. Thus,

$$
\left|t_{j}\right| \leq \frac{1}{2}\left(\frac{|D|}{|d|}+|d|\right) \leq \frac{|D|+1}{2} .
$$

Since $f\left(n_{j}\right)=l_{j} m_{j}^{2}$, so

$$
n_{j}=\left|\frac{-b \pm \sqrt{D+4 a l_{j} m_{j}}}{2 a}\right| \leq \frac{|b|+\left|t_{j}\right|}{2 a} \leq \frac{|b|+(|D|+1) / 2}{2 a}<A_{0} .
$$

This contradicts $n_{j} \geq A_{0}$. Therefore from Theorem 2.2 there are infinitely many $n_{1}$ and $n_{2}$ such that $\lambda\left(f\left(n_{1}\right)\right) \neq \lambda\left(f\left(n_{2}\right)\right)$ and hence $\lambda(f(n))$ changes sign infinitely often.

As we remarked above, one should not expect Theorem 2.3 to be true for polynomials of higher degree.

We prove some partial results of special quadratic polynomials.

Theorem 2.4. Let $f(n)=n^{2}+b n+c$ with $b, c \in \mathbb{Z}$. Suppose there exists a positive integer $n_{0} \geq A_{0}$ (with $\left.a=1\right)$ such that $\lambda\left(f\left(n_{0}\right)\right)=-1$. Then $\lambda(f(n))$ changes sign infinitely often.

Proof. We observe the following identity

$$
f(n) f(n+1)=f(f(n)+n)
$$

which can be verified directly. Hence we have

$$
\lambda(f(n)) \lambda(f(n+1))=\lambda(f(f(n)+n)) .
$$


If $\{\lambda(f(n))\}_{n=1}^{\infty}$ is a constant sequence, then it follows from (2.11) that

$$
\lambda(f(n))=+1, \quad \text { for all } n=1,2, \ldots
$$

Therefore if there is $n_{0} \geq A_{0}$ such that $\lambda\left(f\left(n_{0}\right)\right)=-1$, then by Theorem 2.3. $\{\lambda(f(n))\}_{n=1}^{\infty}$ changes sign infinitely often. This proves the theorem.

The proof of Theorem 2.2 shows that the solvability of the diophantine equation

$$
X^{2}-4 a l Y^{2}=D
$$

is critical in solving the problem. In general, there is no simple criterion to determine the solvability of the equation (2.12) except if we know the central norm of the continued fraction of the irrational number $\sqrt{a l}$. For more discussion in this area, we refer the readers to [6]-[9]. The following theorem deals with a special case of $D$ for which we can solve the equation (2.12).

Theorem 2.5. Let $f(x)=p x^{2}+b x+c$ with prime number $p$. Suppose the discriminant $D=b^{2}-4 p c$ is a non-zero perfect square. Then $\lambda(f(n))$ changes sign infinitely often.

Proof. We first choose positive integers $l_{1}$ and $l_{2}$ such that $p l_{1}$ and $p l_{2}$ are not perfect squares and $\lambda\left(l_{1}\right) \neq \lambda\left(l_{2}\right)$. So the Pell equations

$$
X^{2}-4 p l_{j} Y^{2}=1, \quad j=1,2
$$

have infinitely many positive solutions $(X, Y)$. Let $D=q^{2}$ with $q \geq 1$. Then any positive solution $(X, Y)$ of (2.13) gives a positive solution $(q X, q Y)$ of

$$
X^{2}-4 p l_{j} Y^{2}=D \text {. }
$$

We can choose $X$ large enough so that $-b+q X>0$. On the other hand, we have $X^{2} \equiv 1(\bmod p)$ by (2.13) and $q^{2} \equiv b^{2}(\bmod p)$ because $D=b^{2}-4 p c$. Therefore $(q X)^{2} \equiv b^{2}(\bmod p)$. Since $p$ is a prime, so either (a) $q X \equiv b(\bmod p)$ or (b) $q X \equiv-b(\bmod p)$. We define

$$
n=\frac{-b \pm q X}{2 p}
$$

where the sign \pm is determined according to Cases (a) or (b) so that $n$ is a positive integer. Therefore $(n, q X)$ is a positive solution of the equations $f(n)=l_{j} m^{2}$. Then our theorem follows readily from Theorem 2.2 .

\section{REFERENCES}

[1] P. Borwein, R. Ferguson and M. Mossinghoff, Sign changes in sums of the Liouville function. Math. Comp. 77 (2008), no. 263, 1681-1694.

[2] J. Cassaigne, S. Ferenczi, C. Mauduit, J. Rivat and A. Sarkozy On finite pseudorandom binary sequences IV: The Liouville function II, Acta Arithmetica XCV. 4 (2000) 343-359.

[3] S. Chowla The Riemann Hypothesis and Hilbert's Tenth Problem, Gordon and Breach, New York, 1965.

[4] C. B. Haselgrove A disproof of a conjecture of Pólya, Mathematika 5 (1958), 141-145.

[5] I. Katai, Research Problems in Number Theory II, Ann. Univ. Sci. Budapest. Sect. Comput. 16 (1996), 223-251.

[6] R. Mollin, Central norms and continued fractions, Int. J. Pure Appl. Math. 55 (2009), no. 1, $1-8$.

[7] R. Mollin, Norm form equations and continued fractions, Acta Math. Univ. Comenian. (N.S.) 74 (2005), no. 2, 273-278.

[8] R. Mollin, Criteria for simultaneous solutions of $X^{2}-D Y^{2}=c$ and $x^{2}-D y^{2}=-c$, Canad. Math. Bull. 45 (2002), no. 3, 428-435. 
[9] R. Mollin, The Diophantine equation $a x^{2}-b y^{2}=c$ and simple continued fractions, Int. Math. J. 2 (2002), no. 1, 1-6.

[10] G. Pólya, Verschiedene Bemerkungen zur Zahlentheorie, Jahresber. Deutsch. Math.-Verein. 28 (1919), 31-40.

[11] A. Sarkozy, Unsolved Problems in Number Theory, Periodica Mathematica Hungarica Vol.42 (1-2), (2001), pp 17-35.

[12] C. L. Siegel, Über einige Anwendungen Diophantischer Approximationen, Abh. Preussischen Akademie der Wissenshaften, Phys. Math. Klasse (1929) pp. 41-69.

Department of Mathematics, Simon Fraser University, Burnaby, British Columbia, CANADA V5C 1 S6.

E-mail address: pborwein@sfu.ca

E-mail address: kkchoi@math.sfu.ca

E-mail address: hganguli@sfu.ca 